\title{
Taxa de câmbio, poupança e produtividade: impactos de curto e longo prazo ${ }^{1}$
}

\author{
Paulo Gala ${ }^{2}$ \\ Gilberto Libânio ${ }^{3}$
}

\begin{abstract}
Resumo
O trabalho tem por objetivo analisar, sob o ponto de vista teórico, a relação entre nível da taxa de câmbio e desenvolvimento econômico. A hipótese principal segue a literatura de "doença holandesa" e baseia-se na ideia de que câmbios competitivos contribuem para a formação e manutenção do setor manufatureiro da economia. A existência de retornos crescentes nesse setor influencia fortemente o aumento da produtividade no longo prazo. Discute-se, também, como a manutenção de uma taxa de câmbio competitiva poderá estimular a formação de poupança doméstica ao evitar aumentos artificiais do salário real pela via da sobrevalorização cambial.
\end{abstract}

Palavras-chave: Câmbio; Poupança e Investimento; Desenvolvimento econômico - Brasil.

\begin{abstract}
Exchange rates, savings and productivity: short and long-term impacts

The objective of this paper is to analyze, from a theoretical point of view, the relation between real exchange rates and economic development. The main hypothesis is very much in line with the Dutch disease literature and states that competitive currencies contribute to the existence and maintenance of the manufacturing sector in the economy. This, in turn, brings about higher growth rates in the long run, given the existence of increasing returns in the industrial sector and its importance in generating technological change and increasing productivity in the overall economy. We also show from a theoretical perspective how a competitive exchange rate can stimulate domestic savings by avoiding consumption booms based on currency overvaluation.
\end{abstract}

Keywords: Exchange rate; Savings; Investment; Economic development - Brazil.

JEL F3, F4, O2.

\section{Introdução}

O debate sobre os efeitos da apreciação cambial na economia brasileira continua intenso. Como nos anos 1990, voltam a surgir argumentos favoráveis à apreciação do câmbio real, por exemplo: i) um Real apreciado seria favorável à aquisição de máquinas e equipamentos baratos no exterior, estimulando o investimento agregado; ii) o câmbio apreciado facilitaria a aquisição de tecnologias que não possuímos do exterior. Ambos os argumentos, entretanto,

(1) Trabalho recebido em 17 de outubro de 2008 e aprovado em 11 de março de 2010.

(2) Professor da Fundação Getulio Vargas, São Paulo, SP, Brasil. E-mail: pgala@uol.com.br.

(3) Professor do Centro de Desenvolvimento e Planejamento Regional (Cedeplar) da Faculdade de Ciências Econômicas da Universidade Federal de Minas Gerais (UFMG), Belo Horizonte, MG, Brasil. E-mail: gilberto@cedeplar.ufmg.br

Economia e Sociedade, Campinas, v. 20, n. 2 (42), p. 229-242, ago. 2011. 
parecem não descrever bem o que se observou durante a intensa apreciação cambial brasileira ao longo dos anos 1990, ou seja, baixíssimo nível de investimento agregado e baixo dinamismo tecnológico na indústria brasileira nos setores de ponta, especialmente, se comparados ao que ocorreu no leste da Ásia.

O efeito mais forte da apreciação cambial nas cadeias produtivas de bens comercializáveis, tanto agrícolas quanto industriais, sente-se, sobretudo, nas margens de lucro. A um dado nível de custo em dólares de produtos comercializáveis, a apreciação cambial representa queda imediata e intensa de preços de venda e margens de lucro em reais em toda a cadeia que trabalha com preços internacionais, em particular, nos setores que não têm poder de mercado. A redução do valor de máquinas e equipamentos importados decorrente da apreciação cambial está longe de compensar a redução nos lucros que, baixos, não estimulam o investimento. Os aumentos de importação de bens de capital observados na balança comercial são, primordialmente, direcionados para a substituição da produção de máquinas e equipamentos domésticos o que, por si só, poderá reduzir a capacidade de inovação e melhora tecnológica em setores de ponta. $\mathrm{O}$ aumento da importação de máquinas não significa, necessariamente, um aumento do investimento agregado.

Sobre a aquisição de tecnologia no exterior, vale observar que a apreciação acaba tendo efeito oposto do que argumentam os defensores do câmbio valorizado. Nas cadeias globais de fornecimento, as decisões sobre transferências de tecnologia são feitas pelas multinacionais que alocam sua produção de acordo com a situação de cada plataforma de produção, observando aspectos macroeconômicos, institucionais etc. Regiões ou países que apresentam alta volatilidade cambial e recorrentes ciclos de sobrevalorização, como é o caso do Brasil, acabam ficando fora desse circuito. Não à toa, a Ásia dinâmica consolidouse como grande plataforma de produção industrial para o resto do mundo, apresentando altíssimas taxas de investimento agregado e inovação tecnológica, enquanto a América Latina, com destaque ao Brasil, perdeu um enorme espaço desde os anos 1980.

Como consequência dos efeitos negativos mencionados acima, a apreciação cambial tende a associar-se a baixas taxas de crescimento econômico. Existe, hoje, importante literatura econométrica que procura relacionar taxas de crescimento econômico com a administração cambial e níveis de taxa de câmbio real. Em trabalhos sobre o tema, Cavallo et al. (1990); Dollar (1992); Razin; Collins (1997) encontram uma relação negativa entre sobrevalorização cambial e crescimento econômico para uma longa série de países no período 1970-1990. Quanto mais depreciada a taxa de câmbio, maiores as taxas de crescimento. Acemoglu et al. (2002) percebem evidências semelhantes entre câmbio real e crescimento. Em um trabalho sobre instituições, políticas macroeconômicas e 
crescimento de 96 países, de 1970 a 1997, não se consegue descartar o efeito do câmbio real em variações da taxa de crescimento per capita dos países ao longo do tempo. Rodrik (2007); Fajnzylber et al. (2002) encontram resultados parecidos ao estudar o crescimento comparado das economias latino-americanas e de outros países no período 1960-99.

Apesar dessa crescente literatura empírica, a discussão teórica sobre o tema ainda tem sido pouco desenvolvida. Bresser-Pereira; Nakano (2003); LevyYeati; Sturzenegger (2007) destacam um possível canal de aumento de poupança e investimento dependente de câmbios competitivos. Williamson (2008); Palma (2005); Bresser-Pereira (2007) destacam, também, os efeitos sobre produtividade de níveis de câmbio sobrevalorizados. Como resume Montiel (2008), existem dois principias canais na literatura que têm surgido recentemente para tratar dos efeitos do câmbio no crescimento: i) o canal de aumentos de produtividade ou "TFP enhancing"; e ii) o canal de estímulos a poupança e investimento.

Este trabalho pretende contribuir para a literatura teórica que discute a relação entre taxa de câmbio e crescimento econômico. Tomando como ponto de partida o modelo de Bhaduri; Marglin (1990), são apresentadas aqui perspectivas teóricas que exploram possíveis canais macro dos efeitos da apreciação cambial. Além da breve introdução, o artigo está dividido em outras cinco seções. A um tratará dos efeitos da apreciação cambial nos lucros e salários reais. As seções dois, três e quatro apresentarão perspectivas teóricas sobre os efeitos da apreciação (depreciação) cambial nos níveis de renda, consumo e investimento agregado, poupança interna e externa e produtividade sob uma perspectiva de curto e longo prazo e, na última, as conclusões.

\section{Câmbio real, salários e lucros}

A seção começa com uma análise dos efeitos da apreciação cambial nas margens de lucro agregadas e no salário real ${ }^{4}$. Esta análise parte de uma divisão hipotética da economia em três setores: i) tradables puros, ii) non tradables puros e iii) non tradables com insumos tradables. Inicia-se, portanto, a tradicional regra de formação de preços em ambiente de concorrência imperfeita abaixo:

$$
p_{i}=\left(1+m_{i}\right) w / b
$$

em que $p$ é o nível de preços do setor $i, m$ o nível de mark up do setor $i, b$ a produtividade do trabalho e $w$ o salário nominal. Para o setor de non tradables puro, o nível de preços pnt dependerá do mark up, do salário nominal e da produtividade, todos constantes no curto prazo. Rearranjando-se os termos, o mark

(4) Ver Bresser-Pereira (2006)

Economia e Sociedade, Campinas, v. 20, n. 2 (42), p. 229-242, ago. 2011. 
up, por sua vez, dependerá do salário nominal, da produtividade e nível de preços de non tradables:

$$
\begin{aligned}
& p_{n t}=\left(1+m_{n t}\right) w / b \\
& m_{n t}=\left(b p_{n t} / w\right)-1
\end{aligned}
$$

O mesmo raciocínio poderá ser aplicado ao setor de tradables puro com uma diferença. Para uma economia aberta e relativamente pequena (price taker) os preços de tradables são endógenos e determinados pelo câmbio nominal (e) e preços em dólares $\mathrm{p}^{*}$ :

$$
\begin{aligned}
& p_{t}=\left(1+m_{t}\right) w / b \\
& p_{t}=e p^{*} \\
& m_{t}=\left(b e p^{*} / w\right)-1
\end{aligned}
$$

Uma apreciação cambial reduz o preço de venda dos tradables e, tudo o mais constante (salários nominais, preços externos e produtividade) reduz a margem de lucro do setor de tradables. A apreciação cambial pode, no entanto, aumentar os lucros do setor non tradables que opera com insumos importados (ins*)

$$
\begin{aligned}
& p_{i t}=p_{n t}=\left(1+m_{i t}\right)\left(\beta w / b+(1-\beta)\left(e i n s^{*}\right)\right) \\
& m_{i t}=\left(p_{i t} /\left(\beta w / b+(1-\beta)\left(e i n s^{*}\right)\right)\right)-1
\end{aligned}
$$

No caso do setor de tradables que opera com insumos tradables, uma apreciação cambial sempre reduzirá o mark up, pois a queda no preço de venda tende a ser maior do que a de um componente parcial do custo. O argumento de que o câmbio apreciado é favorável ao investimento agregado por baratear o preço de máquinas e equipamentos poderá ser entendido aqui como uma situação em que o preço reduzido de insumo fará aumentar a margem de lucro para um setor non tradable.

Em termos agregados, o que ocorreria com o nível de mark up? O mark up agregado poderia ser entendido como a média ponderada pelo tamanho de cada setor $(\mathrm{j}, \mathrm{k}, 1)$, logo:

$$
j m_{t}+k m_{n t}+l m_{i t}=m
$$

Uma apreciação cambial reduz o mark up do setor de tradables, mantém inalterado o mark-up do setor de non tradables e aumenta o mark up do setor non tradables com insumos tradables. O efeito final sobre o mark up geral do sistema dependerá do tamanho relativo de cada setor. Se o setor de tradables for maior do 
que o setor de non tradables com insumos tradables $(j>l)$, necessariamente, uma apreciação cambial reduzirá o mark up do sistema. Quanto maior o componente non tradable de custo de produção de tradables, maior será o efeito nos mark ups.

Resta ver como ficam as outras variáveis do sistema como decorrência da apreciação (depreciação) cambial. O nível geral de preços deverá cair (subir), por ser um composto de preço de tradables e non tradables:

$$
p=\alpha e p^{*}+(1-\alpha) p_{n t}
$$

O salário real deverá aumentar (cair), pois é uma relação entre salário nominal e nível de preços:

$$
w / p=w /\left(\alpha e p^{*}+(1-\alpha) p_{n t}\right)
$$

O câmbio real deverá apreciar-se (depreciar-se), na medida em que o câmbio nominal cai (sobe) mais do que o aumento do nível geral de preços que depende, em parte, dos preços de non tradables.

$$
\theta=e p^{*} / p
$$

Por fim, assumindo-se que o setor de tradables é maior do que o de non tradables com insumos importados, o mark up agregado ao sistema deverá cair, ou seja, para dados níveis de produtividade e salário nominal, uma apreciação cambial poderá resultar em redução nas margens de lucro do sistema e aumentos do salário real. As próximas seções discutirão como as dinâmicas de determinação de renda, investimento e poupança são afetadas por essa queda de mark ups no curto e no longo prazo.

\section{Câmbio real e determinação do nível de renda}

Seguimos aqui Bhaduri; Marglin (1990). O modelo parte da definição da poupança agregada $S$, dependendo de uma parcela fixa $s$ do lucro dos capitalistas. Os trabalhadores consomem o total de sua renda, portanto não poupam.

$$
S=s R=s(R / Y)\left(Y / Y^{*}\right) Y^{*}
$$

em que $R$ é a renda dos capitalistas e $Y^{*}$, o produto potencial. Definindo-se $h=R / Y$ como a porcentagem de renda dos capitalistas em relação à renda total, $z=Y / Y^{*}$ como o nível de utilização de capacidade instalada e o produto potencial como $Y^{*}=1$, chega-se a:

$$
\begin{aligned}
& S=\operatorname{sh} z \\
& 1>h>0 \\
& 1>z>0
\end{aligned}
$$

Economia e Sociedade, Campinas, v. 20, n. 2 (42), p. 229-242, ago. 2011. 
Definindo-se $W / Y$ como a participação dos trabalhadores na renda, $N$ como trabalhadores empregados e $1 / b=N / Y$, tem-se que,

$$
W / Y=w N / p Y=1 / b^{*} w / p=1 /(1+m)
$$

e a participação dos capitalistas na renda, $h=R / Y$ será,

$$
h=R / Y=(p Y-w N) / p Y=1-W / Y=m /(1+m)
$$

Levando-se em consideração a hipótese de que os trabalhadores consomem toda sua renda, aumentos dos salários reais significarão reduções da poupança interna e aumentos de consumo. Como consequência, a demanda agregada poderá cair ou aumentar, dependendo dos efeitos da queda das margens de lucro na função investimento.

Tal função do modelo depende, positivamente, da margem de lucro $h$ e do nível de utilização da capacidade instalada $z$ :

$$
I=I(h, z), I_{h}>0, I_{z}>0
$$

Poupança e investimento igualam-se no equilíbrio do mercado de bens em uma economia fechada (que define uma relação IS).

$$
\operatorname{sh} z=I(h, z)
$$

O nível de utilização da capacidade instalada em relação a margens de lucro dependerá da seguinte derivada:

$$
\begin{aligned}
& \partial z / \partial h=\left(I_{h}-s z\right) /\left(s h-I_{z}\right) \\
& I_{h}=\partial I / \partial h>0
\end{aligned}
$$

A partir da condição keynesiana de que o equilíbrio no mercado de bens dá-se por variações do nível de poupança e não de investimento $\left(s h-I_{z}>0\right)$ e como sh é sempre positivo, a utilização da capacidade instalada aumentará ou diminuirá, dependendo da diferença $\left(I_{h}-s z\right)$.

Se o investimento for pouco elástico a variações nas margens de lucro, quedas de salário real terão efeitos recessivos, pois a queda do consumo não será compensada por um aumento de investimento dos empresários $\left(I_{h}<s z\right)$. Essa é a clássica tese do subconsumo, em que reduções de salário real reduzem o consumo e demanda agregada e, nesse caso, o regime de acumulação na economia poderia ser caracterizado como "wage-led". Por outro lado, se os investimentos forem altamente sensíveis à margem de lucro, acontecerá, então, o efeito oposto. Reduções do salário real aumentarão os lucros que, por sua vez, aumentarão a 
demanda agregada e a utilização de capacidade instalada. A economia será, dessa forma, descrita como em um regime de "profit-led growth".

Estendendo o modelo para uma economia aberta, Bhaduri; Marglin (1990) introduzem funções para o volume de exportações $X_{e}$ e importações $X_{m}$ que dependem, respectivamente, do câmbio real $\theta$ e do nível de utilização de capacidade instalada $z$, com as seguintes elasticidades:

$$
\begin{aligned}
& \left(d X_{e} / d \theta\right)\left(\theta / X_{e}\right)=\eta_{e} \\
& \left(d X_{m} / d \theta\right)\left(\theta / X_{m}\right)=-\eta_{m} \\
& \left(\partial X_{m} / \partial z\right)\left(z / X_{m}\right)=u
\end{aligned}
$$

No novo equilíbrio do mercado de bens, o total da poupança mais o gasto com importações $M$ deverão igualar-se ao total do investimento mais o gasto em exportações $E$,

$$
\operatorname{sh} z+M=I(h, z)+E
$$

A derivada parcial de utilização de capacidade instalada em relação à margem de lucro será muita próxima da situação de economia fechada (expressão 19):

$$
\partial z / \partial h=\left(I_{h}-s z\right)\left(g u+s h-I_{z}\right)
$$

em que $g$ representa a participação inicial de importações e exportações sobre o produto e $u$ é a elasticidade do volume de importações em relação à utilização da capacidade instalada. Assumindo-se, outra vez, que $\left(g u+s h-I_{z}\right)>0$, chega-se a conclusões semelhantes às desenvolvidas inicialmente.

No modelo em economia aberta, uma desvalorização real do câmbio reduzirá o salário real e aumentará a margem de lucro dos empresários. Haverá aumento de investimento, exportações e nível de renda, contanto que as funções sejam suficientemente elásticas. No caso das exportações e importações, o efeito total sobre a balança comercial e contas correntes será positivo, se valer a condição Marshall Lerner $\left(\eta_{e}+\eta_{m}>1\right)$.

\section{Câmbio real, poupança externa e poupança interna}

Em sua forma resumida, o modelo mostra que o equilíbrio macroeconômico poderá ser expresso como:

$$
y=C+I+E-M=C(\theta)+I(h(\theta), z)+E(\theta)+M(\theta, z)
$$

Em termos de equilíbrio de poupanças tem-se que, 


$$
S+M-E=I
$$

ou como expresso em (24)

$$
\operatorname{sh}(\theta) z+M(\theta, z)-E(\theta)=I(h(\theta), z)
$$

As trajetórias de poupança interna e externa dependem do câmbio que afeta todas as variáveis acima elencadas. Com funções suficientemente elásticas, um câmbio real mais depreciado poderá aumentar o nível de renda via aumento de exportações, reduzir o nível de consumo, aumentar a poupança interna, reduzir a poupança externa e aumentar o nível de investimento. Teríamos, portanto, o clássico caso asiático cujo crescimento, liderado por investimentos e exportações, resulta em superávits em conta-corrente.

O modelo permite, também, vislumbrar outros tipos de trajetória. Um câmbio sobrevalorizado poderá elevar o nível de renda via aumento do consumo e, dependendo das elasticidades, via aumento do investimento. Nesse tipo de trajetória, o crescimento será, necessariamente, acompanhado de aumento de poupança externa. A situação de déficit em conta-corrente é compatível com crescimento liderado por consumo, eventualmente, por investimentos. Teríamos aqui o padrão mais tradicional observado na América Latina com crescimento e déficits em contas-correntes.

Alguns autores têm argumentado que o modelo asiático não seria implementável no Brasil ou América Latina devido ao baixo nível de poupança privada. Invertendo a causalidade entre déficit em conta-corrente e poupança, os autores argumentam que a baixa propensão individual a consumir dos asiáticos seria responsável pelos altos níveis de poupança interna e superávits em contacorrente. Sem entrar em explicações "culturalistas", o modelo acima exposto endogeniza o consumo como função do salário real que, por sua vez, depende do câmbio real. Assim, o segredo para o alto nível de poupança dos asiáticos estaria, segundo o modelo, nos reduzidos salários reais, dado um nível de produtividade.

No caso latino-americano, as sucessivas crises cambiais dos últimos anos seriam explicadas pelo elevado nível de consumo e excessiva utilização de poupança externa. Tentativas de crescimento puxado pelo consumo a partir de taxas de câmbio real sobrevalorizadas e salários artificialmente elevados resultariam em "insuficiência" de poupança interna e déficit em conta-corrente. O problema latino-americano da "insuficiência de poupança" estaria na sobrevalorização cambial e não, em supostos hábitos de consumo.

Vale notar que toda essa discussão é feita a partir de determinados níveis de produtividade. A elevação do salário real sem contrapartida de aumentos de produtividade é problemática, pois acaba colocando a economia em uma trajetória insustentável que termina em crise de balanço de pagamentos. Por outro lado, em uma situação de câmbio competitivo, há um aumento de lucratividade dos 
investimentos que tende a aumentar o nível de produtividade da economia no longo prazo, permitindo aumentos de salários reais de forma equilibrada. A próxima seção discute em detalhe os impactos do nível do câmbio real na dinâmica de produtividade.

\section{Câmbio real e dinâmica de produtividade}

O nível do câmbio real também tem um papel fundamental na dinâmica macroeconômica a partir de uma perspectiva de longo prazo. Ao influir na determinação da especialização setorial da economia, notadamente no que diz respeito a estímulos à indústria, o impacto do nível do câmbio na dinâmica de produtividade é elevado. Sobrevalorizações cambiais são, em especial, nocivas a processos de desenvolvimento econômico, pois reduzem substancialmente a lucratividade da produção e investimento nos setores de bens comercializáveis manufatureiros. Ao realocar recursos aos setores não manufatureiros, sobretudo à produção de commodities (com retornos decrescentes de escala) e a setores não comercializáveis, as sobrevalorizações cambiais acabam por afetar toda a dinâmica tecnológica da economia. Subvalorizações, por outro lado, estimulam a produção e investimento nos setores manufatureiros em que retornos crescentes de escala são possíveis.

Ao determinar a rentabilidade da produção de manufaturas por meio da relação preço comercializáveis/não comercializáveis, o câmbio real acaba definindo a viabilidade de setores econômicos importantes para o aumento da produtividade geral da economia. Sobrevalorizações podem impedir a transferência de mão de obra dos setores de baixa para os de alta produtividade, já que o preço dos bens não comercializáveis fica, artificialmente, elevado. Um dos canais importantes de progresso técnico e aumento de produtividade acaba assim bloqueado, impedindo a economia de transitar da situação de imaturidade para a maturidade nos termos kaldorianos. Uma moeda competitiva, por outro lado, poderá ser um estímulo adequado para a integração de trabalhadores em atividades de alta produtividade e retornos crescentes.

$\mathrm{Na}$ tradição keynesiana e kaldoriana, a dinâmica tecnológica e a de produtividade dependem fortemente do processo de acumulação de capital e do próprio nível de produção agregado. As inovações tecnológicas estão, em geral, associadas às novas máquinas e equipamentos produzidos, o que significa dizer que processos de crescimento e aumento de demanda, puxados por investimento são acompanhados, de modo geral, por aumentos de produtividade. Nesse sentido, estabelece-se uma relação de causalidade entre a taxa de crescimento da produtividade e a de crescimento da produção em que um aumento na produção, induzido pela demanda, provoca aumento da produtividade em setores nos quais se verifica a presença de economias dinâmicas de escala e "learning-by-doing". Há, portanto, uma relação positiva entre a taxa de crescimento da produtividade do 
trabalho e a de crescimento da produção, conhecida na literatura como "lei de Kaldor-Verdoon". Um aumento da demanda agregada, ao induzir uma aceleração da taxa de crescimento da produção, acaba por acelerar o ritmo de crescimento da produtividade do trabalho.

A correlação entre o crescimento do produto industrial e o desempenho geral da economia poderá ser entendida a partir dos aumentos de produtividade encontrados no setor industrial. Há duas explicações para tal efeito. A primeira diz respeito à transferência de trabalhadores de setores de baixa produtividade (trabalho precário) para atividades industriais que apresentam produtividade elevada. Como há excesso de oferta de trabalho (surplus labor) nos setores tradicionais e de baixa produtividade, a transferência de trabalhadores aos setores modernos tem pouco ou nenhum impacto no nível de produção dos setores tradicionais.

De acordo com Kaldor (1966), esse processo caracteriza a transição das economias da imaturidade para a maturidade, em que imaturidade significa um estado de permanente oferta de trabalho nos setores de trabalho precário e subsistência, portanto de baixa produtividade. A segunda razão para a correlação entre o produto da indústria $\mathrm{e}$ o aumento da produtividade relaciona-se à existência de retornos crescentes de escala estáticos e dinâmicos em atividades manufatureiras como mencionado acima. Retornos estáticos dizem respeito a economias de escala encontradas dentro das firmas e retornos dinâmicos referemse a aumentos de produtividade derivados de "learning by doing", externalidades positivas e "spill-overs" tecnológicos.

Existe outra forma de abordar os efeitos do nível do câmbio real na especialização setorial da economia. Uma proposição básica do modelo de crescimento com restrições do balanço de pagamentos de Thirlwall (1979) é o crescimento de longo prazo, restrito pelo equilíbrio externo da economia. Sob algumas hipóteses simplificadoras, mostra-se que a taxa de crescimento de longo prazo de uma economia é dada pela relação entre o crescimento das exportações $(x)$ e a elasticidade-renda das importações $(\pi)$, a chamada lei de Thirlwall:

$$
y_{B P}=\frac{x}{\pi}
$$

Nesse caso, é possível argumentar que a elasticidade-renda das importações não é independente do padrão de especialização da economia na medida em que diferentes setores apresentam respostas distintas em termos de importação quando a economia cresce. Esse argumento foi desenvolvido formalmente por Araújo; Lima (2007) a partir de um modelo multissetorial inspirado em Pasinetti (1981) em que elasticidades-renda agregadas das importações e exportações dependem das elasticidades setoriais e da participação de cada setor nos totais de importação e exportação. Levando em consideração tal 
resultado juntamente à Lei de Thirlwall, pode-se concluir que o padrão de especialização de uma economia afeta sua taxa de crescimento restrita pelo balanço de pagamentos ${ }^{5}$. Na abordagem aqui apresentada, a política cambial pode ter efeitos duradouros na composição setorial da economia, afetando, portanto, as elasticidades-renda das importações e exportações, com efeitos positivos ou negativos sobre o crescimento de longo prazo. Taxas de câmbio competitivas podem estimular o setor industrial e a diversificação produtiva, impactando de forma favorável as elasticidades de longo prazo da lei de Thirlwall, tanto de exportações quanto de importações. Ao contrário, "a prolonged period of exchange-rate appreciation can be detrimental for growth in the long run, because it may induce a change in the structural determinants of the balance of payments (BoP) constraint" (Barbosa-Filho, 2006, p. 3).

Uma política cambial adequada pode ajudar a estimular o setor não tradicional de bens comercializáveis da economia, especialmente, o de manufaturas para exportação. Como argumenta Williamson (2008) em seu "development approach" para a política cambial, uma taxa de câmbio competitiva é fundamental para estimular o desenvolvimento do setor de bens comercializáveis não dependentes de commodities, evitando assim os problemas da doença holandesa (ver Bresser-Pereira, 2007) e desindustrialização (ver Palma, 2005). Ao estimular a produção de manufaturas para o mercado mundial, uma taxa de câmbio competitiva pode ajudar os países a escalar a escada do desenvolvimento tecnológico ${ }^{6}$.

\section{Conclusões}

Os argumentos apresentados nas seções anteriores procuraram discutir os impactos do nível do câmbio real nos salários reais, taxas de lucro, investimento agregado, poupança externa e interna, produtividade, portanto no crescimento. As primeiras seções trataram dos efeitos de curto prazo do nível do câmbio real na lucratividade das empresas e nos níveis de renda, consumo, poupança e investimento agregado. Em casos de sobrevalorização excessiva, a redução de margens de lucro nos setores de produção de bens comercializáveis significará uma queda nos níveis de investimento, a não ser que o nível de utilização da capacidade instalada mais do que compense a redução das margens de lucro.

(5) Sobre modelos teóricos que discutem a relação entre padrão de especialização, crescimento e taxa de câmbio, ver também Cimoli (1992); Pugno (1996); Blecker (1999).

(6) Cabe notar que a análise aqui desenvolvida não trata do papel do governo e do sistema financeiro na dinâmica da economia. Embora reconhecendo a importância dessas variáveis, entende-se que tal discussão ultrapassa o escopo deste trabalho, cujo objetivo central é contribuir teoricamente para a relação entre câmbio e crescimento, tomando como ponto de partida o modelo Bhaduri-Marglin (1990) e a literatura sobre doença holandesa.

Economia e Sociedade, Campinas, v. 20, n. 2 (42), p. 229-242, ago. 2011. 
Para os setores produtores de bens não comercializáveis ou de comercializáveis maquiladores, a apreciação cambial poderá significar um aumento de investimento na medida em que reduzirá preços de insumos importados sem reduzir preços de venda, ou seja, aumentará as margens de lucro com possíveis impactos positivos para o investimento. É importante notar que, embora o investimento agregado da economia aumente por conta desse efeito, poderá haver uma especialização setorial perversa no sentido de restrições do balanço de pagamentos e capacidade de geração de divisas. Se grande parte do investimento estiver concentrada no setor produtor de bens não comercializáveis, haverá alta probabilidade de restrição de divisas para o crescimento no longo prazo. Relativa depreciação cambial, por outro lado, incentivará uma especialização setorial com foco na produção de manufaturas para o mercado mundial e doméstico devido ao efeito de aumento de rentabilidade. Os setores maquiladores sofrerão e os setores produtores de não comercializáveis com insumos importados terão suas margens reduzidas. Mudanças no nível da taxa de câmbio têm, portanto, fortes efeitos no modelo, em termos de dinâmicas setoriais da economia.

Como argumentado na seção dois do trabalho, o efeito líquido final da apreciação do câmbio no investimento agregado dependerá dos mark ups relativos de cada setor e do nível de utilização da capacidade instalada. Para um dado nível de atividade econômica, tal investimento estará sujeito, no modelo, ao mark up geral do sistema. Este, por sua vez, dependerá do tamanho relativo do setor de bens comercializáveis em relação ao tamanho do setor de bens não comercializáveis que utiliza insumos comercializáveis. Em uma economia com o primeiro setor maior do que o segundo, apreciações cambiais significarão, necessariamente, reduções de margens gerais de lucro. Conforme o modelo, com tudo mais constante, o investimento agregado deverá, então, cair. As seções três e quatro do trabalho analisaram tais efeitos de forma mais detalhada.

A penúltima seção do trabalho discutiu efeitos do câmbio real na produtividade de uma perspectiva de longo prazo. O nível do câmbio real importa não só como estímulo permanente ao aumento de exportações, dado um nível de renda mundial, mas também como determinante da especialização setorial da economia. Ao promover a industrialização de setores domésticos, um câmbio competitivo contribui fortemente para aumentos de produtividade ao acionar os efeitos kaldorianos mencionados acima (economias de escala, "learning by doing", retornos crescentes etc.). Poderá contribuir ainda para a superação das restrições externas ao crescimento, na medida em que reduz a propensão da economia como um todo a importar e estimular indústrias domésticas cuja demanda externa apresenta-se de forma mais dinâmica (não commodities).

Em relação ao caráter regressivo de políticas cambiais que estimulem o aumento de competitividade da moeda, cabe ressaltar o papel de políticas 
compensatórias utilizadas no curto prazo para compensar a queda relativa do salário real. Por outro lado, tentativas de aumento do salário real pela via da sobrevalorização cambial, geralmente, têm-se mostrado bastante problemáticas. Os inúmeros casos de populismo cambial e crise na América Latina, recentemente, são testemunhas disso. A via por excelência de aumento sustentado do salário real passa pela redução do desemprego e incorporação dos trabalhadores nos setores dinâmicos da economia mundial como bem mostraram as experiências asiáticas de sucesso. Uma política de câmbio competitivo tem exatamente esse objetivo. Ao promover a competitividade das indústrias domésticas, contribui para a mudança estrutural da economia que, em última análise, causa o desenvolvimento econômico.

Uma das principais mensagens do trabalho sugere que os impactos da política cambial no investimento agregado, poupança externa e interna, produtividade e crescimento sejam fortes e duradouros no curto e no longo prazo.

\section{Referências bibliográficas}

ACEMOGLU, D.; SIMON, J.; JAMES R. Institutional causes, macroeconomic symptoms: volatility, crisis and growth. Cambridge, MA: MIT, 2002.

ARAÚJO, R.; LIMA, G. A structural economic dynamics approach to balance-ofpayments constrained growth. Cambridge Journal of Economics, 31, 2007.

BARBOSA-FILHO, N. Exchange rates, growth and inflation. Apresentado na II Annual Conference on Development and Change ACDC, Campos do Jordão, SP, Brazil, Nov. 2006

BHADURI, A.; MARGLIN, S. Unemployment and the real wages: the economic basis for contesting political ideologies. Cambridge Journal of Economics, 14, Dec. 1990.

BLECKER, R. Kaleckian macro models for open economies. In: DEPREZ, J.; HARVEY, J. (Ed.). Foundations of international economics: post Keynesian perspectives. London: Routledge, 1999.

BRESSER-PEREIRA, L. C. Exchange rate, fix, float or manage it? Preface to Mathias Vernengo (Ed.). Financial integration or dollarization: no Panacea. Cheltenham: Edward Elgar, 2006.

. The Dutch disease and its neutralization, a Ricardian approach. Revista de Economia Política, São Paulo, v. 28, n. 1, jan./mar. 2007.

; NAKANO. Crescimento com poupança externa? Revista de Economia Política, São Paulo, v. 23, n. 2, abr.jun. 2003.

CAVALlO, D.; COTTANI, J. A.; KAHN, M. S. Real exchange rate behaviour and economic performance in LDCs. Economic Development and Cultural Change, 39, p. 6176, Oct. 1990.

DOLLAR, D. Outward-oriented developing economies really do grow more rapidly: evidence from 95 LDCs, 1976-1985. Economic Development and Cultural Change, 40, p. 523-544, 1992. 
CIMOLI, M. Exchange rate and productive structure in a technological gap model. Economic Notes by Monte dei Paaschi di Siena, v. 21, n. 3, 1992.

EICHENGREEN, B. The real exchange rate and economic growth. UC Berkeley, 2007. Mimeografado.

FAJNZYLBER, P.; LOAYZA, N.; CALDERÓN, C. Economic growth in Latin America and the Caribbean: stylized facts, explanations and forecasts. Washington, DC: The World Bank, 2002.

FRENKEL, R. Real exchange rate and employment in Argentina, Brazil, Chile and Mexico. Buenos Aires: Cedes, 2004. Paper presented to the G24. Disponível em: http://www.unctad.org/templates/Page.asp?intItemID=1751\&lang=1 / http://policydialogue.org/files/events/Frenkel Exchange Rate_Employment.pdf. Acesso em: jul. 2005.

; TAYLOR, Lance. Real exchange rate, monetary policy and employment. New York: United Nations, 2006. (Desa Working Paper, n. 19).

KALDOR, Nicholas. Causes of the slow rate of economic growth of the United Kingdom. In: FURTHER essays on economic theory. New York: Holmes \& Meier Publisher, 1966.

1978.

. Further essays on economic theory. New York: Holmes \& Meier Publisher,

LEVY-YEYATI, E.; STURZENEGGER, F. Fear of floating in reverse: exchange rate policies in the 2000s. Harvard: Kennedy School of Government, 2007. Mimeografado.

MONTIEL, P.; SERVEN, L. Real exchange rate, savings and growth: is there a link? The Washington, DC: World Bank, 2008. (Policy Research Working Paper).

PALMA, G. Four sources of de-industrialization and a new concept of the Dutch Disease. In: OCAMPO, J. A. (Ed.). Structural dynamics and macroeconomic theory. Palo Alto: Stanford University Press, 2005.

PASINETTI, L. Structural change and economic growth - a theoretical essay on the dynamics of the wealth of nations. Cambridge: Cambridge University Press, 1981.

PUGNO, M. A Kaldorian model of economic growth with labour shortage and major technical changes. Structural change and economic dynamics, 7, 1996.

RAZIN, O.; COLLINS, S. Real exchange rate misalignment and growth. Forthcoming in: RAZIN, Assaf; SADKA, Efraim (Ed.). International economic integration: public economics perspectives. Cambridge University Press / NBER Working Paper n. 6147, 1997.

RODRIK, D. Real exchange rate and economic growth: theory and evidence. John F. Kennedy School of Government, Harvard University, Draft, Jul. 2007.

THIRLWALL, A. P. The balance of payments constraint as an explanation of international growth rates differences. Banca Nazionale del Lavoro Quarterly Review, v. $128,1979$.

WILLIAMSON, J. Exchange rate economics. Washington, DC: Peterson Institute for International Economics, 2008. (Working Paper Series, 08/3). Disponível em: http://www.iie.com/publications/interstitial.cfm?ResearchID=884. Acesso em: jul. 2005. 